\title{
The Mechanistic Study on the Effect of Acacia concinna and Cymbopogon nardus on Lipid Metabolism
}

\author{
Wijitrapha Ruangaram ${ }^{1 *}$, Eisuke Kato ${ }^{2}$
}

\author{
${ }^{1}$ Division of Applied Bioscience, Graduate School of Agriculture, Hokkaido University, Sapporo, Hokkaido 060-8589, \\ Japan,wijit@chem.agr.hokudai.ac.jp \\ ${ }^{2}$ Division of Fundamental AgriScience and Research, Research Faculty of Agriculture, Hokkaido University, Sapporo, \\ Hokkaido060-8589, Japan, eikato@chem.agr.hokudai.ac.jp \\ *Corresponding author. Email: wijit@chem.agr.hokudai.ac.jp
}

\begin{abstract}
Obesity is one of the most concerning health problems globally. At the moment, medicinal plants have been widely studied in order to assist in the treatment of obesity instead of the developed drugs. From our previous study, Thai medicinal plants were tested through screening methods regarding anti-obesogenic activity and several candidates were selected. Subsequently, in this study, 2 of the candidate plants naming Acacia concinna (Wild.) DC., and Cymbopogon nardus (L.) Rendle were selected and studied in more detail on the mechanistic aspect related to lipid metabolism. The plants were extracted in 50\% (v/v) aq. methanol and resuspended in 10\% DMSO to be tested in 3T3-L1 adipocytes and NCTC 1469 hepatocytes. The cells were treated with the plant extract and the effect on expression of lipid metabolism related genes were examined using RT-qPCR. The target genes related to lipid metabolism are listed as following: adipogenesis (PPAR $\gamma, \mathrm{C} / \mathrm{EBP} \alpha$ ), lipogenesis (FAS, ACC-1, SREBP-1), lipolysis (HSL, ATGL, perilipin-1) and fatty acid $\beta$-oxidation (PPAR $\alpha$ ). In 3T3-L1 adipocytes, $A$. concinna plants downregulated adipogenesis genes, lipogenesis genes, and lipolytic genes. On the other hand, $C$. nardus has a difference in some genes such as upregulated C/EBPo and no significant changes in ATGL. Furthermore, In NCTC 1469 hepatocytes, 2 plants acted distinctively in each gene. A. concinna lowered all the target gene expressions with some being significantly different but some were not. $C$. nardus downregulated gene expression of $\mathrm{C} / \mathrm{EBP} \alpha$, FAS, SREBP-1, HSL, ATGL and PPAR $\gamma$ but no significant changes were observed in ACC-1 and PPAR $\gamma$. A. concinna and C. nardus might have potential to combat obesity through improving the lipid metabolism, which can act as anti-adipogenic and anti-lipogenic agents. Further study such as in vivo study will be conducted to strengthen the data.
\end{abstract}

Keywords: Lipid metabolism, Mechanistic study, Thai medicinal plants.

\section{INTRODUCTION}

Obesity has been a worldwide concern in the past few years. It is a health state that excessive lipids accumulate in the body [1]. It can lead to numerous diseases including hypertension, dyslipidemia, coronary heart disease and so on. Therefore, the prevention or the treatment of obesity is necessary.

There are several ways to treat obesity. This includes the use of commercial drugs. The examples of commercial drugs are benzphetamine and phendimetrazine [2]. These reduce the food consumption by promoting satiety or suppress an appetite, which will result in prevention of weight gain. Since the restriction of foods by oneself is quite difficult, people tend to be more reliant on the drugs without knowing that there might be some detrimental effects. The detrimental effects normally take place when the drugs are used for a long term. It can vary from mild effects such as insomnia, headache, constipation to severity like cardiovascular and psychiatric side effects [3]. Hence, there is a limitation to the usage of the anti-obesity commercial drugs.

Meantime, an alternative method such as the traditional remedy is taken into consideration. One of the common methods in traditional remedies is the consumption of medicinal plants. In parallel to a study to develop drugs, an interest in the use of medicinal plants is also growing among the researchers. Even though the medicinal plants generally show a lower efficacy than drugs, there are lower side effects as well [4]. Therefore, 
medicinal plants have a merit to be used for a longer period.

In our previous study, Thai medicinal plants were tested for an anti-obesogenic potential and candidates to be used for prevention of obesity are selected [5]. Acacia concinna (Wild.) DC and Cymbopogon nardus (L.) Rendle were two of the selected plants with a potential to inhibit pancreatic lipase, enhance lipolysis and reduce lipid accumulation in adipocytes. Hence, in this study, the effect of these 2 medicinal plants were studied in more detail regarding the adipogenesis, lipid metabolism (lipogenesis, lipolysis) and fatty acid $\beta$-oxidation gene expression in adipocytes and hepatocytes

The body text starts with a standard first-level heading like INTRODUCTION or any other heading suitable to the content and context. First level headings are in all caps. Copy the content and replace it for other first-level headings in remaining text. Reference citations should be within square bracket [1]. Headings should always be followed by text.

\section{MATERIALS AND METHOD}

\subsection{Preparation of Medicinal Plant Extracts}

Thai medicinal plants were purchased from a local store in Samutprakan, Thailand. The plant samples were stored at the Ministry of Public Health, Department of Thai Traditional and Alternative Medicine (Thailand). The voucher specimens (authentic crude drug) were issued for use as medicinal plants' species references. The voucher specimens of A.concinna and $C$. nardus are WR063, 10000621 and WR037, 10000595, respectively. The plants were ground into fine particles and $5 \mathrm{~g}$ of each plant were soaked in $50 \%(\mathrm{v} / \mathrm{v})$ aqueous methanol for 3 days. The suspension of each plant was filtered through a filter paper. The filtrates were evaporated to obtain the extracts which will be used in further experiment.

\subsection{Cell Culture}

\subsubsection{T3-L1 Adipocytes}

The 3T3-L1 cells were cultured and differentiated as described in previous study with a slight modification [5]. On day 6, the cells were washed with phosphate buffered saline (PBS) and then serum starved overnight. Afterwards, the extracts were diluted to desired concentrations with Dulbecco's modified Eagle's medium (DMEM) without phenol red and added to the cells. After 24 hrs incubation, the cells were harvested.

\subsubsection{NCTC 1469 Hepatocytes}

The NCTC 1469 (JCRB9075) were obtained from the Japanese Collection of Research Bioresources Cell Bank (Osaka, Japan). The cells were cultured in 6-well plates using DMEM containing 10\% (v/v) horse serum. The cells were allowed to proliferate for 3 days and then the extracts diluted with the medium were added to the cells. The cells were incubated overnight and then harvested

\subsection{RNA extraction and Quantitative Real Time PCR}

Total RNA was extracted from the harvested cells ( $n=6)$ using ReliaPrep ${ }^{\mathrm{TM}}$ RNA Cell Mini System kit (Promega, Tokyo, Japan). Procedure followed the kit instruction except before adding 2-propanal, the lysate was passed through a gauge syringe needle no.22 (Terumo Corporation, Tokyo, Japan) and centrifuged at $15,000 \times \mathrm{g}, 3$ mins. The obtained total RNA was used to synthesize a complementary DNA (cDNA) using ReverTra Ace ${ }^{\mathrm{TM}}$ qPCR RT Master Mix kit (Toyobo, Tokyo, Japan) according to the manufacturer's procedure. The qPCR was performed on Takara Thermal Cycle Dice Real Time system (Takara Bio Inc., Shiga, Japan) with KAPA SYBR FAST Universal reagent (Sigmaaldrich, Tokyo, Japan). The relative gene expression was calculated using cycle threshold method $\left(2^{-\Delta \Delta c t}\right)$ and normalized with YWHAZ reference gene.

\subsection{Statistical Analysis}

Statistical analysis was performed using GraphPad Prims 9 software (GraphPad Software, San Diego, CA, USA). Statistical analysis of difference between the sample group and control group was determined by student $\mathrm{t}$ test. Data are expressed as mean \pm standard deviation $(n=6)$. $p$-values $<0.05\left(^{*}\right)$ and $p$-values $<0.01$ $(* *)$ were considered statistically significant.

\section{RESULTS AND DISCUSSION}

\subsection{Effect of Extracts on Lipid Metabolism Related Gene Expression in 3T3-L1 Adipocytes}

The target genes which regulate adipogenesis and lipid metabolism were tested in 3T3-L1 adipocytes. Followings are target genes in each process: adipogenesis (C/EBP $\alpha$, PPAR $\gamma$ ), lipogenesis (FAS, ACC-1, SREBP-1) and lipolysis (HSL, ATGL, PLIN-1). The result showed that the 2 medicinal plants acted differently (Figure 1).

A. concinna downregulates all the target genes (Figure 1A, B). C/EBP $\alpha$ and $\operatorname{PPAR} \gamma$ are the major regulators of adipogenesis which handles the expression of lipogenesis and lipolysis genes [6]. Therefore, downregulation of lipogenesis (FAS, ACC-1, SREBP-1) and lipolysis (HSL, ATGL, PLIN-1) genes are presumed to occur through the reduced expression of $\mathrm{C} / \mathrm{EBP} \alpha$ and PPAR $\gamma$. This result indicates that $A$. concinna extract targets $\mathrm{C} / \mathrm{EBP} \alpha, \mathrm{PPAR} \gamma$ or a more upstream regulator like $\mathrm{C} / \mathrm{EBP} \beta$. 
(A)

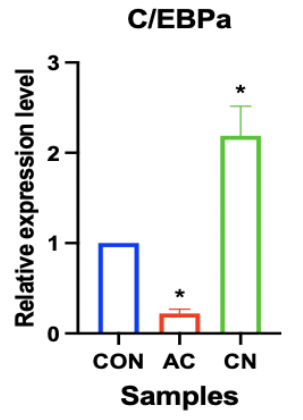

(E)

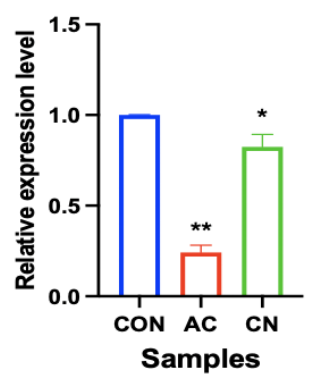

(B)

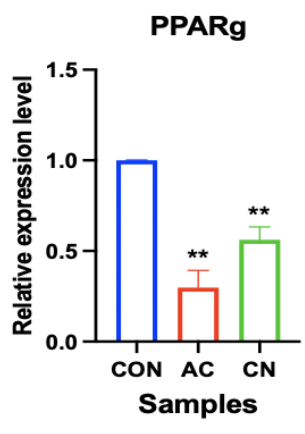

(F)

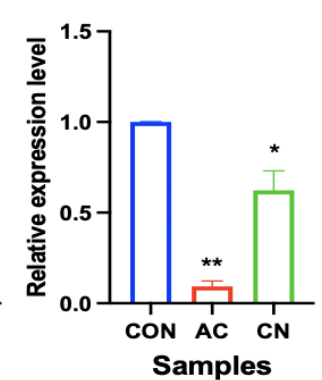

(C)

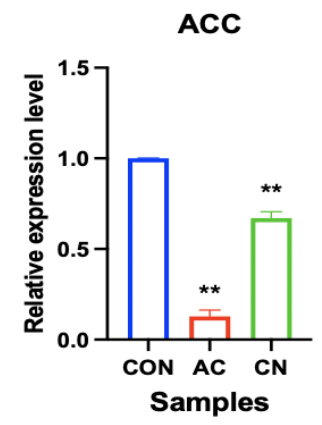

(G)

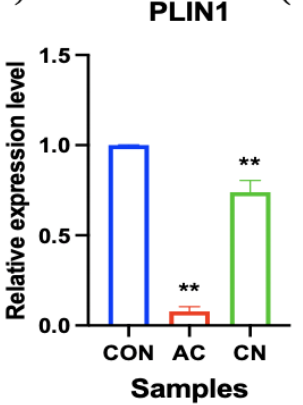

(D)
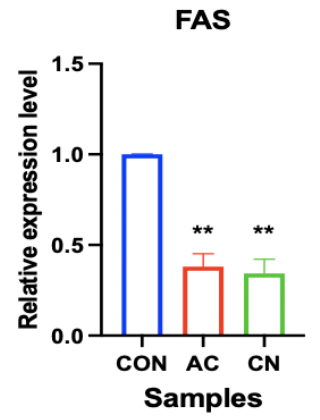

(H)

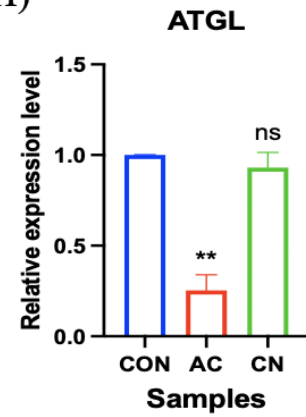

Figure 1. lipid metabolism related target genes in 3T3-L1 adipocytes. Control used here is $0.1 \%$ DMSO-treated cells. Data were expressed as mean \pm standard deviation $(\mathrm{n}=6)$. $p$-values $<0.05(*), p$-values $<0.01(* *)$ were considered as statistically significant.

The results of A. conccina against 3T3-L1 adipocytes also gives a clear mechanistic insight to the previous observation that $A$. concinna reduces lipid accumulation [7]. A decreased level of lipogenesis genes will reduce lipid synthesis and thus reduce the lipid amount in the cells. A. concinna also induces lipolysis in 3T3-L1 adipocytes and reduced expression of lipolysis gene might be a conflicting result. However, we have already shown that lipolysis induction by A. concinna occurs through activation of PKA and ERK. Activation of lipolysis through the signalling pathway generally occurs much faster than the reduction of protein level. Thus, the observed downregulation of lipolytic genes do not immediately influence the lipolytic activity of the cell.

C. nardus downregulated PPAR $\gamma$ but upregulated $\mathrm{C} / \mathrm{EBP} \alpha$ (Figure 1A and B). Downregulated PPAR $\gamma$ indicates that $C$. nardus also lowers the lipogenesis and lipolytic genes since PPAR $\gamma$ is a key regulator of lipid metabolism [8]. As with this observation, most of the target genes were downregulated by $C$. nardus. However, expression of ATGL was unchanged, and the decrease of other genes were relatively mild compared to A. concinna. This is probably due to upregulation of $\mathrm{C} / \mathrm{EBP} \alpha . \mathrm{C} / \mathrm{EBP} \alpha$ together with $\mathrm{PPAR} \gamma$ are known to regulate expression of lipid metabolism and thus upregulation of $\mathrm{C} / \mathrm{EBP} \alpha$ may result in milder downregulation of target genes.
From these findings, $C$. nardus might focus on different targets from A. concinna which affect adipogenesis and its downstream processes. AMPK signalling pathway is one of our speculations. AMPK can regulate both adipogenesis and lipogenesis [9]. The report stated that the phosphorylation of AMPK inhibits adipogenesis and lipogenesis through the inhibition of SREBP-1 which leads to the downregulation of adipogenic genes as well as its lipogenic downstream regulators. Hence, $C$. nardus might induce the phosphorylation of AMPK which leads to the inhibition of SREBP-1. Thus, further study is required to determine the target of $C$. nardus which might act differently from A. concinna.

According to our previous observation, C. nardus increased lipolysis but had a scarce effect on reducing the lipid accumulation in 3T3-L1 adipocytes. From the current result of gene expression analysis, lipogenic genes were downregulated by the treatment of $C$. nardus. However, ACC, the enzyme involved in the rate limiting step of lipid synthesis, showed milder downregulation compared to $A$. concinna. This may be the reason why $C$. nardus did not efficiently reduce lipid accumulation. On the other hand, this milder downregulation has the possibility to enhance lipolysis. PLIN1 is known to regulate the access of lipase to lipid droplets. Decreased PLIN1 together with unchanged ATGL and mildly decreased HSL might contribute to the enhanced lipolysis 
by $C$. nardus. However, the plant may also stimulate lipolysis through activation of PKA or ERK, and therefore this part remains as an unclear point.

According to these observations, A. concinna and $C$. nardus was both found to downregulate PPAR $\gamma$ and downregulated lipogenic and lipolytic genes, but their activity to reduce lipid accumulation of 3T3-L1 adipocytes are different presumably due to a distinct effect on $\mathrm{C} / \mathrm{EBP} \alpha$.

\subsection{Effect of Extracts on Lipid Metabolism Related Gene Expression in NCTC 1469 Hepatocytes}

The same target genes with 3T3-L1 adipocytes are selected except for PLIN-1 which is not expressed in the liver [9]. An additional gene PPAR $\alpha$ which regulates fatty acid $\beta$-oxidation and also regulates several genes related to lipolysis and lipogenesis in liver were also examined [10].

The results showed that $C$. nardus downregulated $\mathrm{C} / \mathrm{EBP} \alpha$ whereas there is no significant difference in $A$. concinna-treated cells (Figure 2A). On the other hand, $A$. concinna downregulated PPAR $\gamma$ whereas there is no significant difference in $C$. nardus-treated cells (Figure 2B). These indicate that both of the plants have a possibility to downregulate hepatic lipogenesis.
Downstream of C/EBP $\alpha$ and PPAR $\gamma$ is SREBP-1 and SREBP-1 regulates expression of ACC-1 and FAS which are responsible for lipogenesis [11]. SREBP-1 is downregulated by $C$. nardus and not significant but tends to downregulate by $A$. concinna (Figure $2 \mathrm{E}$ ). FAS is downregulated by both the plants, but ACC is significantly downregulated only by $A$. concinna (Figure $2 \mathrm{C}$, D). These results essentially follow the results against 3T3-L1 adipocytes where these plants could reduce lipid accumulation. Downregulation of both ACC and FAS by A. concinna would result in lipid accumulation reduction in hepatocytes, as with the 3T3$\mathrm{L} 1$ adipocyte result. $C$. nardus, showed no change in ACC but can possibly reduce hepatic lipid accumulation since there is a report stating that decreased FAS without significant change in ACC-1 is enough to reduce lipogenesis in the liver [12]. Furthermore, there is also another possibility that the plants might act on different regulators rather than SREBP-1 to regulate ACC and FAS. For instance, Carbohydrate response element binding protein (ChREBP), which is the transcription factor that can regulate de novo lipogenesis through the expression of lipogenic genes [13]. The report revealed that the sole activation of SREBP-1 could not modulate the expression of lipogenic genes. Thus, further research is required to conclude if these plants have lipogenesis inhibition in the liver as well as its responsible target.
(A)

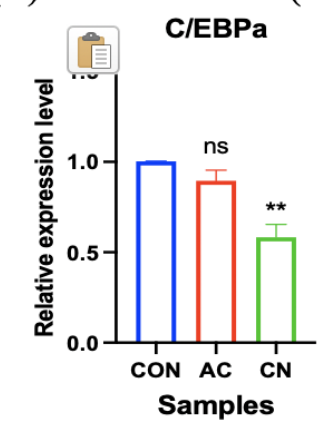

(E)

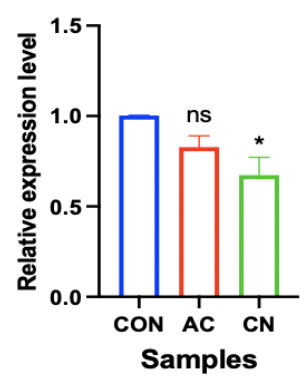

(B)

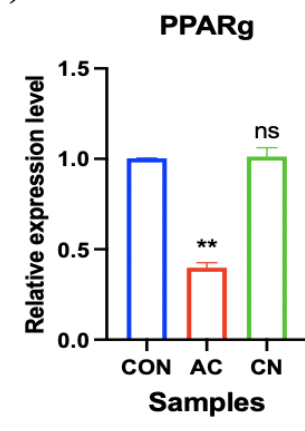

(F)

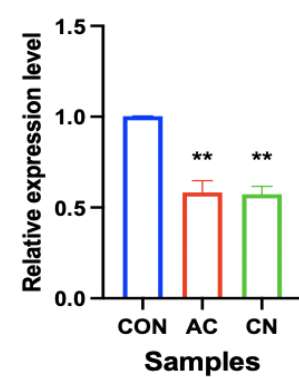

(C)

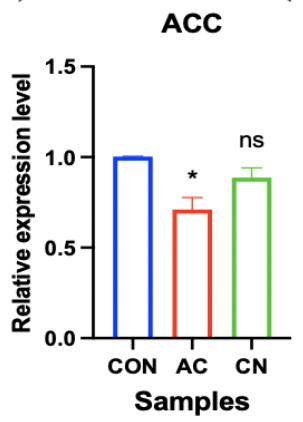

(G)

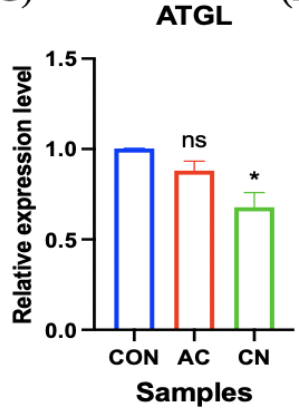

(D)

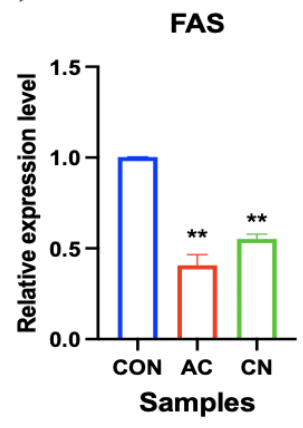

$(\mathrm{H})$

PPARa

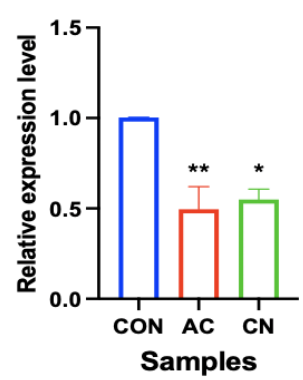

Figure 2. lipid metabolism related target genes in NCTC 1469 hepatocytes. Control used here is $0.1 \%$ DMSOtreated cells. Data were expressed as mean \pm standard deviation $(\mathrm{n}=6)$. $p$-values $<0.05(*), p$-values $<0.01(* *)$ were considered as statistically significant. 
For lipolysis and $\beta$-oxidation related genes, both the plants either downregulated or had no effect (Figure 2F$\mathrm{H})$. These indicate that the plants have no effect or decrease the lipid consumption process. However, decreased lipid synthesis may lead to a decreased lipid consumption process (since there will be not much lipid to use).

According to these results against NCTC 1469 cells, A. concinna and $C$. nardus may have an effect to reduce lipid accumulation in hepatocytes, however further examination is required to conclude this.

\section{CONCLUSION}

To our knowledge, this is the first mechanistic study regarding lipid metabolism in both adipocytes and hepatocytes by 2 medicinal plants, A. concinna and $C$. nardus. The 2 plants were initially found to possess potent anti-obesogenic activity and selected as a candidate to prevent/treat obesity in our previous study. The current result revealed that these 2 medicinal plants show anti-obesogenic activity through down-regulation of adipogenic and lipogenic genes. Further study such as in vivo and/or further in-depth in vitro experiments are warranted to exhibit the possibility of these 2 medicinal plants as an alternative therapeutic approach for antiobesity treatment.

\section{REFERENCES}

[1] E H. Ito, K. Nakasuga, A. Ohshima, Y. Sakai, T. Maruyama, Y. Kaji, et al., Excess accumulation of body fat is related to dyslipidemia in normal-weight subjects, International Journal of Obesity, 28(2) (2004) 242-7.

[2] M.O. Dietrich, T.L. Horvath, Limitations in antiobesity drug development: the critical role of hunger-promoting neurons, Nature Reviews Drug Discovery, 11(9) (2012) 675-91.

[3] J.G. Kang, C.-Y. Park, Anti-Obesity Drugs: A Review about Their Effects and Safety, Diabetes Metab J, 36(1) (2012) 13-25.

[4] Y. Liu, M. Sun, H. Yao, Y. Liu, R. Gao, Herbal Medicine for the Treatment of Obesity: An Overview of Scientific Evidence from 2007 to 2017, Evidence-Based Complementary and Alternative Medicine, (2017).

[5] W. Ruangaram, E. Kato, Selection of Thai Medicinal Plants with Anti-Obesogenic Potential via In Vitro Methods, Pharmaceuticals, 13(4) (2020) 56.

[6] Y.R. Choi, J. Shim, M.J. Kim, Genistin: A Novel Potent Anti-Adipogenic and Anti-Lipogenic Agent, Molecules, 25(9) (2020) 2042.
[7] Z. Zhuoyue, W. Ruangaram, E. Kato, Saponins are responsible for the anti-obesogenic activity of Acacia concinna, J Nat Med, (2021).

[8] E. Rosen, C.-H. Hsu, X. Wang, S. Sakai, M. Freeman, F. Gonzalez, et al., $\mathrm{C} / \mathrm{EBP} \alpha$ induces adipogenesis through PPAR $\gamma$ : A unified pathway, Genes \& development, 16(2002) 22-6.

[9] S. Wei, S. Liu, X. Su, W. Wang, F. Li, J. Deng, et al., Spontaneous development of hepatosteatosis in perilipin-1 null mice with adipose tissue dysfunction, Biochimica et Biophysica Acta (BBA) - Molecular and Cell Biology of Lipids, 1863(2) (2018) 212-8.

[10] M. Yoon, The role of PPAR $\alpha$ in lipid metabolism and obesity: Focusing on the effects of estrogen on PPAR $\alpha$ actions, Pharmacological Research, 60(3) (2009) 151-9.

[11] S. Schadinger, N. Bucher, B. Schreiber, S. Farmer, PPAR 2 regulates lipogenesis and lipid accumulation in steatotic hepatocytes, American journal of physiology Endocrinology and metabolism, 288(2005) E1195-205.

[12] F. Oriente, S. Cabaro, A. Liotti, M. Longo, L. Parrillo, T.B. Pagano, et al., PREP1 deficiency downregulates hepatic lipogenesis and attenuates steatohepatitis in mice, Diabetologia, 56(12) (2013) 2713-22.

[13] P. Ferré, F. Foufelle, Hepatic steatosis: a role for de novo lipogenesis and the transcription factor SREBP-1c, Diabetes, Obesity and Metabolism, 12(s2) (2010) 83-92. 